\title{
Omnidirectional antireflective properties of porous tungsten oxide films with in-depth variation of void fraction and stoichiometry
}

\author{
Nikolaos Vourdas ${ }^{\text {a }}$, Konstantinos Dalamagkidis ${ }^{\mathrm{b}}$, Ioannis Kostis ${ }^{\mathrm{a}}$, Maria Vasilopoulou ${ }^{\mathrm{a}, *}$, \\ Dimitrios Davazoglou ${ }^{\mathrm{a}}$ \\ a NCSR Demokritos, Institute of Microelectronics, Terma Patriarchou Gregoriou, 15310 Athens, Greece \\ ${ }^{\mathrm{b}}$ Institut für Informatik VI, Technische Universität München (TUM), Boltzmannstraße 3, 85748 Garching bei München, Germany
}

\section{A R T I C L E I N F O}

\section{Article history:}

Received 21 June 2012

Received in revised form

26 July 2012

Accepted 1 August 2012

Available online 25 August 2012

\section{Keywords:}

Tungsten oxide

Hot wire deposition

RCWA

Antireflective coatings

\begin{abstract}
A B S T R A C T
We report on the fabrication of porous hot-wire deposited $\mathrm{WO}_{x}\left(\right.$ hwWO $\mathrm{W}_{x}$ ) films with omnidirectional antireflective properties coming from in-depth variation of both (i) void fraction from $0 \%$ at the $\mathrm{Si}$ substrate $/ \mathrm{hwWO}_{x}$ interface to $30 \%$ within less than $7 \mathrm{~nm}$ and to higher than $50 \%$ at the hwWO $/$ air interface, and (ii) $x$, namely hwWO $\mathrm{WO}_{x}$ stoichiometry, from 2.5 at the $\mathrm{Si} / \mathrm{hwWO}_{x}$ to 3 within less than $7 \mathrm{~nm}$. hwWO $\mathrm{W}_{x}$ films were deposited by means of hw deposition at rough vacuum and controlled chamber environment. The films were analyzed by Spectroscopic Ellipsometry to extract the graded refractive index profile, which was then used in a rigorous coupled wave analysis (RCWA) model to simulate the antireflective properties. RCWA followed reasonably the experimental reflection measurements. Void fraction and $x$ in-depth variation, controlled by the hw process, greatly affect the antireflective properties, and improve the omnidirectional and broadband characteristics. The reflection suppression below $10 \%$ within the range of $500-1000 \mathrm{~nm}$ for angles of incidence up to more than $60^{\circ}$ is demonstrated.
\end{abstract}

(c) 2012 Elsevier B.V. All rights reserved.

\section{Introduction}

In the last years the research activity in the area of solar cells has increased and has brought many solutions in order to achieve optimized efficiencies. To approach an optimal performance and to maximize efficiency broadband, antireflective coatings (ARC) are necessary [1].

The standard double layer antireflection coatings typically have a bandwidth lower than one octave, e.g., 400-700 nm [2], while solar cells require an antireflection coating operating in a broader range (e.g., $400-1700 \mathrm{~nm}$ ) [3]. Thin films exhibiting thickness-graded refractive index profiles are being intensively studied recently, for applications in antireflective coatings with enhanced omnidirectionality and broadband characteristics [4]. The refractive index profile and hence the reflection suppression, is controlled either by depositing layers of different materials or by controlling the in-depth void fraction variation using multiple deposition steps, by employing various types of lithography and subsequent pattern transfer such as plasma etching, by incorporating particles and properly tuned deposition processes etc

\footnotetext{
* Corresponding authors.

E-mail addresses: nvourdas@imel.demokritos.gr (N. Vourdas), mariva@imel.demokritos.gr (M. Vasilopoulou).
}

[5-11]. Even though most of these coatings exhibit good AR performance, the scale-up and the integration of most of these processes are either difficult or subject to serious economic constraints.

Recently we reported a simple method for depositing metal oxide films with their stoichiometry depending on the composition of the deposition ambient. It was found before that highly porous, stoichiometric $\left(\mathrm{hwWO} \mathrm{WO}_{3}\right)$ films were deposited at 0.1 Torr of $\mathrm{N}_{2}$. Due to their high porosity these films exhibited refractive index depending on thickness (i.e., deposition time) varying between 2 for thinner films and 1.2 for thicker ones, within the visible range [12]. By switching the deposition ambient from $\mathrm{N}_{2}$ to $\mathrm{H}_{2}$, sub-stoichiometric films, described as hwWO $\mathrm{W}_{x}$ with $x<3$, were also obtained, with their degree of stoichiometry $(x)$ and refractive index depending on the exact $\mathrm{H}_{2}$ partial pressure [13-15].

The aim of this work is to synthesize thin hwWO $\mathrm{W}_{x}$ films with improved omnidirectional AR performance, using hot-wire deposition, which is an easily implemented method as described before. In particular, here we present for the first time the fabrication of porous tungsten oxide films with graded refractive index, by varying the deposition ambient which initially contains $\mathrm{H}_{2}$ and then switched to $\mathrm{N}_{2}$ and we study their reflection performance. It is also shown that these films exhibit controllable in-depth profile of refractive index stemming from both the 
variation of stoichiometry and that of void fraction. The antireflective performance of thin and ultra-thin $\mathrm{hwWO}_{x}$ films on Si and the significant reflection suppression, below $10 \%$, within the broad range of $500-1000 \mathrm{~nm}$, is presented.

\section{Experimental}

\subsection{Tungsten oxide films fabrication}

Depositions of hwWO $\mathrm{Wilms}_{x}$ were carried out in a hot-wire deposition system by heating the $\mathrm{W}$ filament in an ambient containing $\mathrm{N}_{2}$ and $\mathrm{H}_{2}$. The system was containing a stainless steel vacuum chamber (reactor) in which the substrate was positioned on a copper susceptor $5 \mathrm{~cm}$ below the heated W filament. An AC $(50 \mathrm{~Hz})$ current was used to heat the filament, and the temperature was monitored in situ, in order to calibrate electric current vs. filament temperature variation. Gases were injected into the reactor by a showerhead placed above the substrate; the corresponding flows were controlled by a gas line containing mass flow controllers (MFCs), valves and tubing. The pressure was monitored by a capacitance manometer (baratron), whose signal was used as input to a PC and the last was automatically driving a throttling valve placed between the chamber and the pumping system. The tungsten filament was heated at temperatures of $920 \mathrm{~K}, 1020 \mathrm{~K}$ and $1070 \mathrm{~K}$. These temperatures are relatively low, compared to works from other groups, thus allowing only the surface oxide layer to be evaporated and not the metallic W. This feature provides additional advantages that are presented and discussed elsewhere [16]. Si pieces with dimensions of $2 \times 2 \mathrm{~cm}^{2}$ cut from $\left(\begin{array}{lll}1 & 0 & 0\end{array}\right)$ Si wafers were used as substrates. Before deposition the substrates were given a piranha clean, washed in ultra-pure water, dried in a nitrogen stream and loaded into the chamber. After loading, the chamber was evacuated below $1.33 \mathrm{~Pa}$ and then the pressure was stabilized to $133.3 \mathrm{~Pa}$ with the aid of a flow of $\mathrm{H}_{2}$ or $\mathrm{N}_{2}$ while keeping the throttling valve fully open. After pressure stabilization the filament was turn on. Deposition time was used to control film thickness and the times used varied between $1 \mathrm{~s}$ and $60 \mathrm{~s}$. Due to the short deposition times and the high thermal mass of the copper susceptor, the substrate temperature was remaining near room temperature during deposition. After turning-off the filament, samples were left to cool down to room temperature while the pressure and the ambient used for the deposition were maintained. The cooling down was enduring several minutes.

\subsection{Film optical and morphological characterization}

Deposited $\mathrm{hwWO}_{x}$ films were characterized with a variety of techniques including spectroscopic ellipsometry (SE) within the $350-1000 \mathrm{~nm}$ range using a J.A Woolam Inc. M2000F rotating compensator ellipsometer ( $\mathrm{RCE}^{\mathrm{TM}}$ ) running the WVASE32 software at an angle of incidence (AOI) of $75.14^{\circ}$.

The unmonochromatized $\mathrm{Mg} K_{a}$ line at $1253.6 \mathrm{eV}$ (15 keV with $20 \mathrm{~mA}$ anode current) and an analyzer (Leybold EA-11) pass energy of $100 \mathrm{eV}$, giving a full width at half maximum, FWHM, of $1.3 \mathrm{eV}$ for the Au $4 \mathrm{f}_{7 / 2}$ peak, were used in all X-ray photoelectron (XPS) spectra measurements. The oxide stoichiometry was estimated using the XPS-measured $\mathrm{W} 4 \mathrm{f}$ and $\mathrm{O}$ 1s core-level spectra. To this extent, the areas under the photoemission peaks were integrated by fitting the $\mathrm{O} 1 \mathrm{~s}$ and $\mathrm{W}$ 4f spectra with asymmetric Gaussian-Lorentzian curves.

A LEO Supra 35 VP scanning electron microscope (SEM) was used to characterize films morphology.

Specular optical reflectivity measurements were performed using the optical reflectometer FR-Basic by ThetaMetrisis S.A. (without integrating sphere) at near to normal AOI, using Tungsten white light source and spectrometer, and running the FR-Monitor software. An Al mirror with protective coating from Edmund Optics was used for reflection calibration.

\section{Results and discussion}

Tungsten oxide films were deposited with the hot-wire (hw) method using either $\mathrm{N}_{2}$ or $\mathrm{H}_{2}$ environment or a combination of both (switching the one after the other). Anticipating for
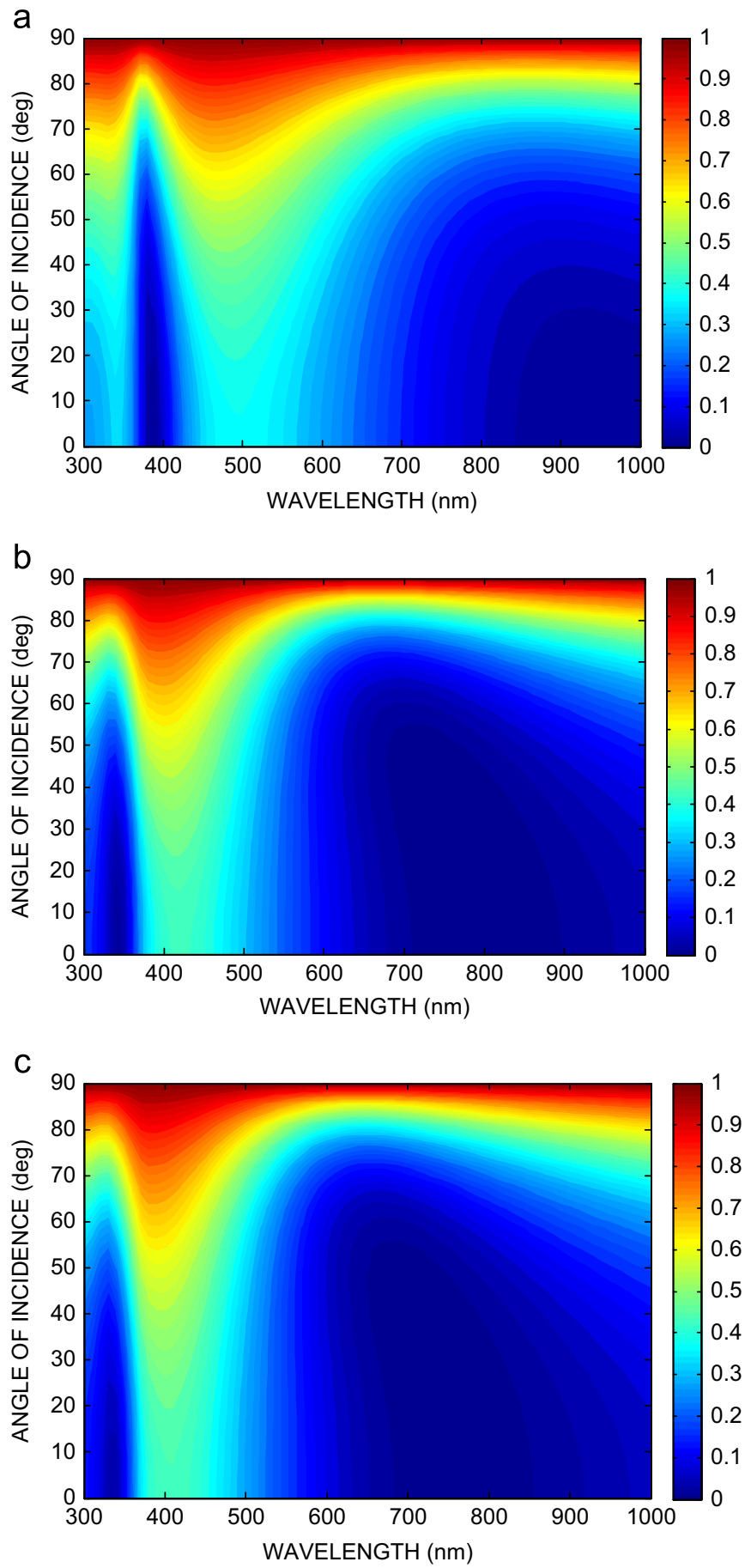

Fig. 1. Reflection calculated from RCWA for a hwWO $/ \mathrm{Si}$ system with hwWO thickness in all cases equal to $107 \mathrm{~nm}$. (a) Dense, non-porous stoichiometric hwWO hwWO $\mathrm{W}_{x}$ with graded refractive index profile as presented in Fig. 5(a). The total area on the figures for which reflection is below $10 \%$ increases from approximately $17 \%$ in (a) to $35 \%$ in (b) and to $37 \%$ in (c). 
modeling details presented next, the calculated reflection of a $\mathrm{Si}$ substrate covered with a $107 \mathrm{~nm}$ thick layer of hwWO $\mathrm{W}_{x}$ and for Angles of Incidence (AOI) varying between 0 and $90^{\circ}$ is shown in Fig. 1. Calculations were based on the rigorous coupled wave analysis (RCWA), and we focus in the region of $500-1000 \mathrm{~nm}$ which is of great importance, for example, for $\mathrm{Si}$ solar cells. Results in Fig. 1(a) pertain to a homogeneous, stoichiometric and compact layer of $\mathrm{hwWO}_{3}$ on $\mathrm{Si}$ and demonstrates that reflection values below $10 \%$ exhibit only for wavelengths above $800 \mathrm{~nm}$ and AOIs of up to $30^{\circ}$ for approximately a region of $17 \%$ of the total area in Fig. 1(a). This low reflection (reflection $<10 \%$ ) area increases to $35 \%$ for a porous $\mathrm{hwWO}_{3}$ film exhibiting 30\% porosity as seen in Fig. 1(b). Finally, a graded refractive index profile (see Fig. 5(a) for the detailed profile), causes a further increase of this area to $37 \%$ by improving the omnidirectionality by $3-4^{\circ}$ and shifting the low reflection region towards lower wavelengths. In the case of inverse pyramid-like textured $\mathrm{Si}$ substrate, routinely used in Si solar cells manufacturing, an ARC coating such as the one described above will cause further decrease of the reflection seen in Fig. 1(b) and (c), as, e.g., shown for the case of $\mathrm{TiO}_{2}$ ARC [11]. For cells operating under suntracking this will decrease the requirements of the tracker for precision and therefore its price, which is related to the total price of the electricity produced.

In order to tune the refractive index profile, depositions of hwWO $\mathrm{W}_{x}$ films were carried out in an ambient initially containing $\mathrm{H}_{2}$ and then switched to $\mathrm{N}_{2}$, in order to controllably change films stoichiometry. Also, different deposition times were used. X-ray photoelectron spectroscopy (XPS) has been applied to follow films stoichiometry. The evolution of the oxide reduction was monitored by measuring the intensity of the W $4 \mathrm{f}$ core levels and calculating the ratio between the $\mathrm{W}^{6+}$ component versus the $\mathrm{W}$ components with lower oxidation states (e.g., 5+, 4+). By depositing the first layers of the film (e.g., the first $7 \mathrm{~nm}$ ) under $\mathrm{H}_{2}$ environment it was found that they were sub-stoichiometric $\mathrm{WO}_{x}$ (i.e., metallic [14]) with $x=2.5$ and their refractive index equal to 3 . Then the deposition ambient was switched to $\mathrm{N}_{2}$ resulting in the deposition of a ca. $100 \mathrm{~nm}$ gradually porous hwWO $\mathrm{WO}_{3}$ layer. In Fig. 2(a) and (b) the XPS W $4 \mathrm{f}$ core levels of hwWO $\mathrm{W}_{x}$ layers deposited under $\mathrm{N}_{2}$ and $\mathrm{H}_{2}$ environment, respectively, are presented. The raw data after a Shirley background subtraction were fitted by a non linear least square routine using peaks with a mix of Gaussian and Lorentzian shape. In Fig. 2(a) the decomposition of W4f photoemission peak was performed using two equal width peaks ( 1 and 2 ) with binding energy of $\mathrm{W}_{4 \mathrm{f}_{7 / 2}}$ centered at $36.0 \pm 0.1 \mathrm{eV}$. The spin orbit splitting of the doublet is $2.13 \mathrm{eV}$ (and a peak ratio of $4: 3$ ) and a third broad peak of $5 p_{3 / 2}$ is located at approximately $42 \mathrm{eV}$. The position and shape of these peaks are representative of $\mathrm{W}$ atoms with an oxidation state $6+$, as expected for $\mathrm{WO}_{3}$ [17]. For the substoichiometric film, the W 4 f peak (Fig. 2(b)) becomes broader indicating that new spectral features appear. A decomposition of this spectrum is shown in Fig. 2(b). A new doublet was added (4 and 5) with spin-orbit splitting and peak ratio fixed at $2.13 \mathrm{eV}$ and $4: 3$, respectively, with all peak widths equal to the peak width of the $W 4 f_{7 / 2}$ in Fig. 2(a). The binding energy of $W 4 f_{7 / 2}$ of the new doublet is at $34.8 \mathrm{eV}$ and is attributed to the presence of $\mathrm{W}^{5+}$ atoms in $\mathrm{WO}_{3-x}$ [18]. The relative atomic concentrations in the analyzed region were calculated by dividing the total peak area of $\mathrm{W} 4 \mathrm{f}$ and $\mathrm{O} 1 \mathrm{~s}$ (not shown here).

The surface atomic composition of the fully oxidized film as derived from the XPS measurements is: W: $1,0: 3.1( \pm 0.1)$ and of the reduced film: $W: 1,0: 2.5( \pm 0.1)$. It should be mentioned that similar results for the reduced films composition were taken when either $\mathrm{H}_{2}$ or forming gas was flowing into the chamber during film deposition. These results show that a compound with composition described by the formula $\mathrm{WO}_{2.5}$ is formed. In this way hwWO $\mathrm{W}_{x}$ films with thickness-graded refractive index profiles with various thicknesses were synthesized. In Fig. 3 the crosssection (left) and top-view (right) scanning electron microscopy (SEM) images of these films are shown.

Similarly, another sample with total thickness of $78 \mathrm{~nm}$ was deposited. In order to extract the refractive index profile multiple depositions were carried out at various film thicknesses. In this way, a resolution of $2 \mathrm{~nm}$ was obtained for the first and top ca. $10 \mathrm{~nm}$ and of $10 \mathrm{~nm}$ for the remaining layer in between the interfaces. The reflection properties of these films at various AOI were calculated by means of RCWA and compared to experimental optical reflection measurements made at near to vertical incidence only. The details on optical measurements are given below. The deposited $\mathrm{hwWO}$ films were characterized optically by means of SE. The beam spot projected on the sample sizes $2 \mathrm{~mm}$ long and therefore is in no case comparable to the characteristic diameter of the nanosized surface characteristics of the hwWO . SE has been previously implemented to examine reflection properties at various interfaces such as $\mathrm{Si} / \mathrm{SiO}_{2}$ [19], indium-tin-oxide (ITO) [20] and $\mathrm{SiN}_{x}$ [21] ARCs.

As reported before, in order to extract the refractive index profile many samples with various thicknesses were deposited. So, after $1 \mathrm{~s}$ of deposition SE analysis yielded the refractive index of hwWO $(x=2.5)$. In order to analyze the sample deposited in $2 \mathrm{~s}$ we used a double layer model, with the lower layer consisting of $\mathrm{hwWO}_{2.5}$. In order to investigate the presence of $\mathrm{hwWO} \mathrm{W}_{2.5}$,
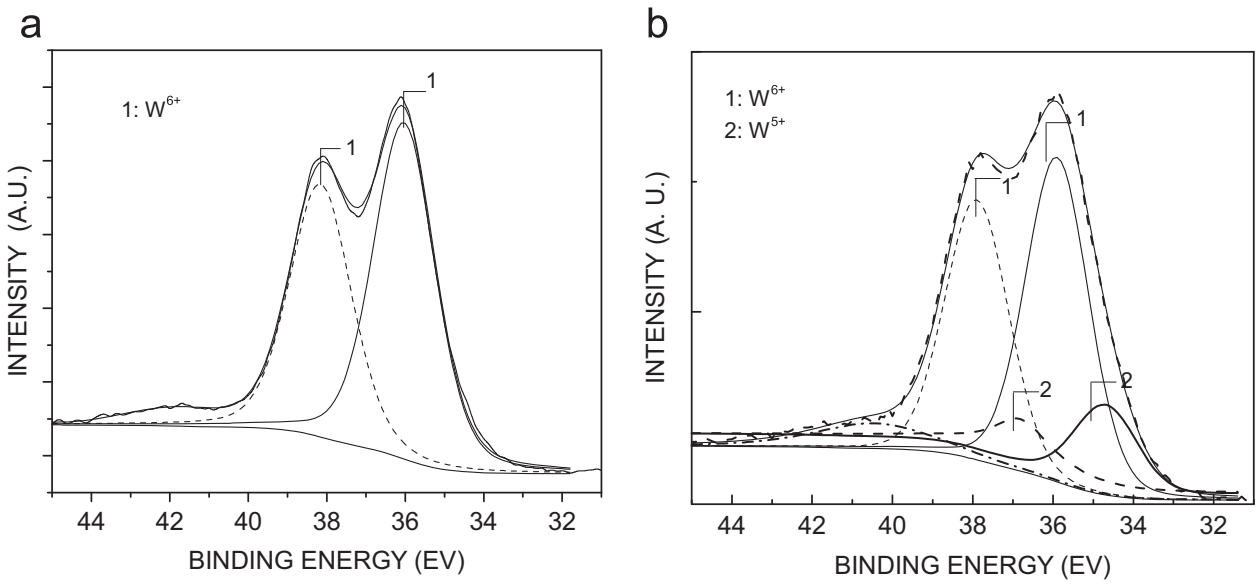

Fig. 2. X-ray photoelectron spectra of $\mathrm{W} 4 \mathrm{f}$ core levels of (a) stoichiometric hwWO 3 and (b) substoichiometric hwWO $\mathrm{W}_{2.5}$. 

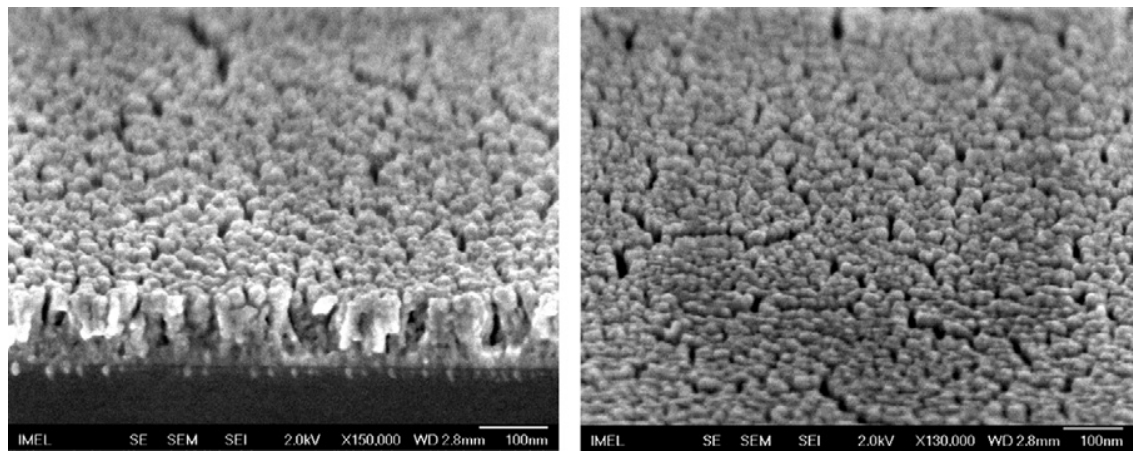

Fig. 3. SEM images of the $107 \mathrm{~nm} \mathrm{hwWO}$ stoichiometric film used for this study in (left) cross-section and (right) top-view.
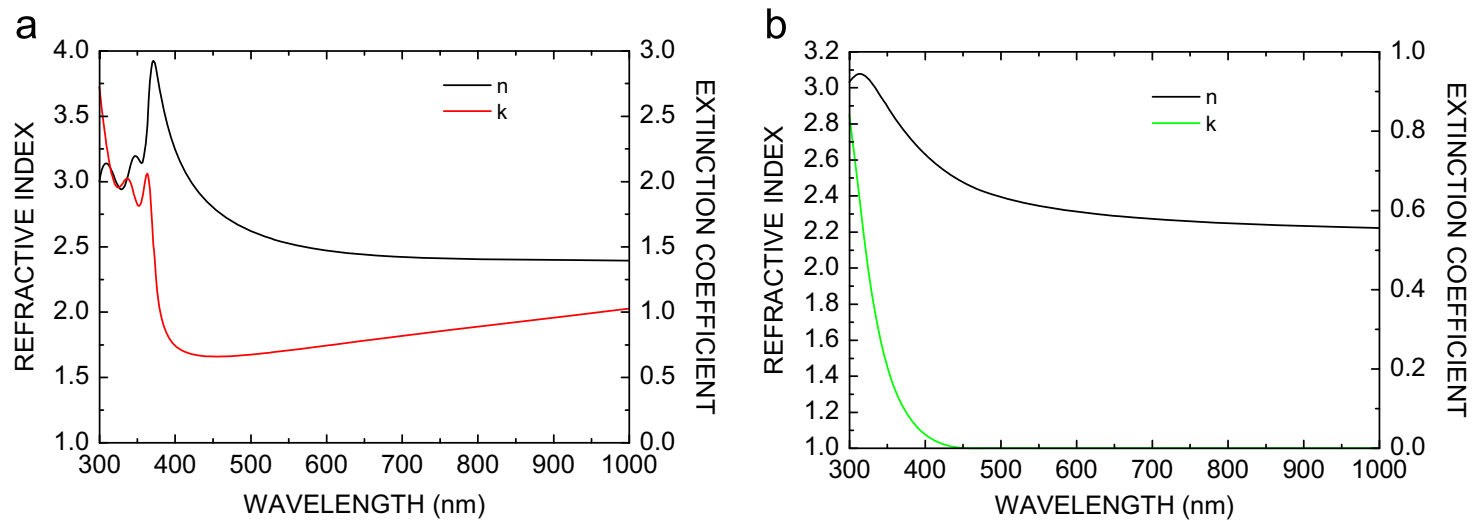

Fig. 4. Refractive index and extinction coefficient dispersion of (a) hwWO $\mathrm{W}_{2.5}$ (sub-stoichiometric) and (b) hwWO $\mathrm{h}_{3}$ (stoichiometric) used for this study.

a

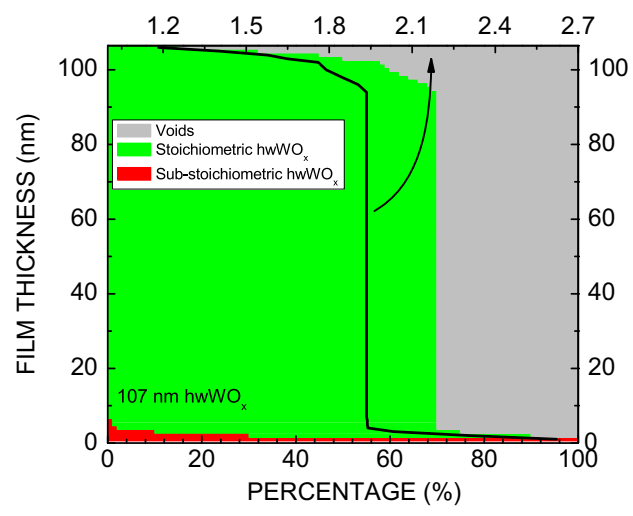

b

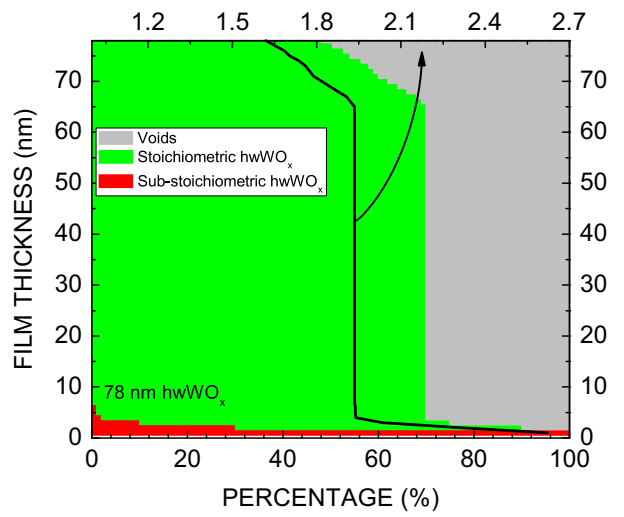

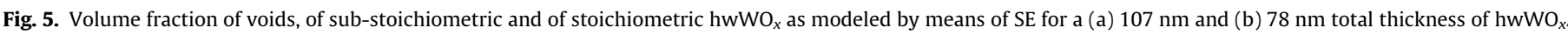
The respective in-depth variation of the effective refractive index (real part) @500 nm is also depicted.

hwWO $\mathrm{WO}_{3}$, and voids in the upper layer, its optical properties were modeled with the aid of the Effective Medium Analysis (EMA) considering a mixture of the above and we extracted the corresponding volume fractions $\left(f_{1}(z), f_{2}(z)\right.$ and $\left.f_{3}(z)\right)$. The next sample was deposited at $5 \mathrm{~s}$ and was considered as a three-layer system the lower one consisting of $h w W_{2.5}$, the intermediate one composed as described previously and the third one was again modeled using the EMA and the constituents considered for the intermediate layer and the volume fractions were extracted again. This procedure was followed for thicker films until reaching the final film thickness ( $78 \mathrm{~nm}$ and $107 \mathrm{~nm}$ ).

The effective refractive index of each layer $\tilde{N}(z)=n-i k$, with $i=\sqrt{-1}$, is then approximated by the formula $\tilde{N}(z)=\left[f_{1}(z) \tilde{N}_{1}^{q}+f_{2}\right.$ $\left.(z) \tilde{N}_{2}^{q}+\left(1-f_{1}(z)-f_{2}(z)\right) \tilde{N}_{3}^{q}\right]^{1 / q}$, with $q=2 / 3 \quad[22]$. The optical properties of the sub-stoichiometric $\left(\tilde{N}_{1}\right)$ and stoichiometric
$\left(\tilde{N}_{2}\right)$ hwWO that used in this study are shown in Fig. 4(a) and (b) while for the voids $\tilde{N}_{3}=1$.

The $\tilde{N}(z)$ (only the real part) at $500 \mathrm{~nm}$ for a $107 \mathrm{~nm}$ and $78 \mathrm{~nm}$ thick hwWO ${ }_{x}$ is shown in Fig. 5(a) and (b). Note that with this approach there is no need to assume a model for the geometry of the surface (composed of conical, pyramid, parabolic-like features) [23].

The fraction of the sub-stoichiometric $h w W_{2.5}$, as seen in Fig. 5(a) and (b), starts from $100 \%$ and goes to zero within less than $7 \mathrm{~nm}$, while at the same time the fraction of (stoichiometric) hwWO $\mathrm{Wr}_{3}$ gradually increases, along with the void fraction. This variation smoothes out the refractive index difference at the $\mathrm{Si}$ $/$ hwWO ${ }_{x}$ interface (at $500 \mathrm{~nm}$ Si: $n=4.29$, Substoichiometric hwWO $: n=2.63$, stoichiometric hwWO $: n=2.39$ ) and therefore suppresses the reflection at this interface. The formation 


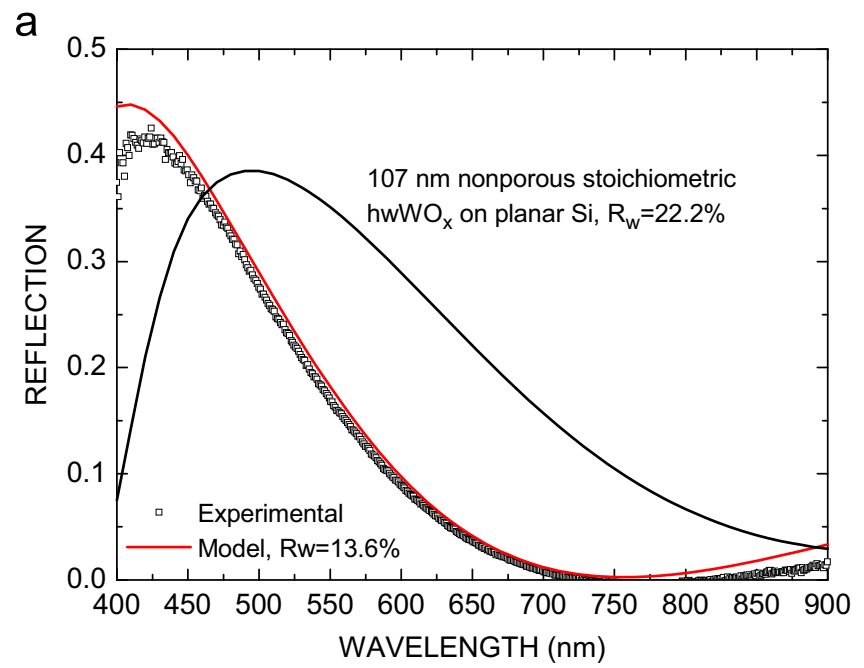

b

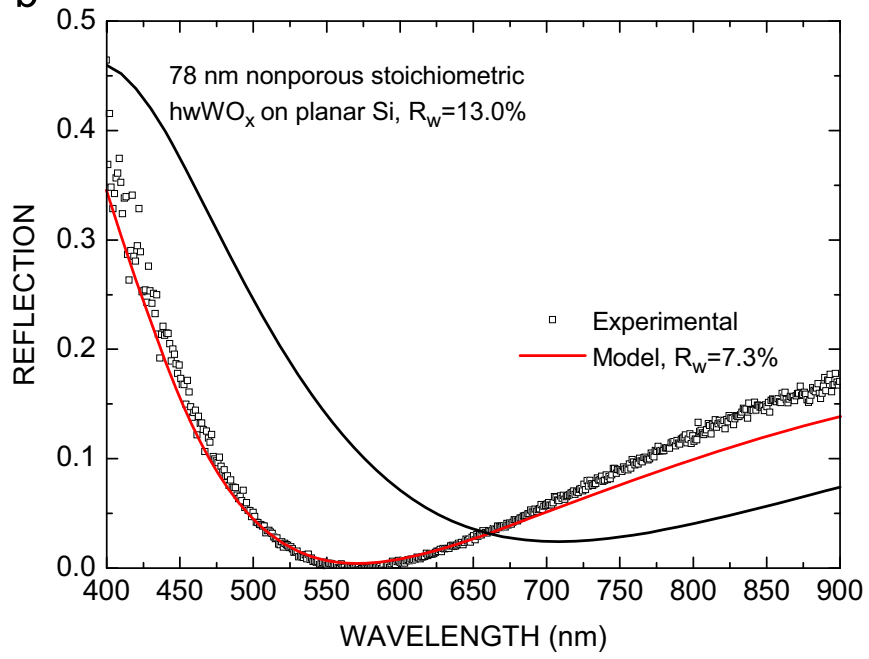

Fig. 6. Experimentally recorded and RCWA-calculated reflection for a (a) $107 \mathrm{~nm}$ and (b) $78 \mathrm{~nm}$ thick hwWO $\mathrm{W}_{x}$ film on $\mathrm{Si}$ wafer. Reflection for equally thick nonporous stoichiometric $\mathrm{hwWO}_{3}$ films from RCWA are also illustrated for comparison purposes. Weighted average reflection for each case $\left(R_{w}\right)$ is also depicted.

mechanism for this gradual decrease of sub-stoichiometric hwWO $\mathrm{W}_{x}$ fraction vs. film thickness, under reducing ambient is discussed elsewhere [13]. The void fraction of $\sim 30 \%$ is kept essentially constant until the final ca. $10 \mathrm{~nm}$, after which point the void fraction increases towards higher values ( $80 \%$ for the $107 \mathrm{~nm}$ film and $50 \%$ for the $78 \mathrm{~nm}$ film), resulting in a corresponding reduction of refractive index.

In Fig. 6 the experimentally measured reflection (with FR-Basic by ThetaMetrisis) for $\mathrm{hwWO}_{x}$ films of thickness $107 \mathrm{~nm}$ and $78 \mathrm{~nm}$ is presented for wavelengths between $400 \mathrm{~nm}$ and $900 \mathrm{~nm}$.

RCWA [24] was used to model reflection at vertical incidence (which corresponds to the first row of data at $\mathrm{AOI}=0$ of Fig. 1 (c). In-depth variation in fraction of voids, $\mathrm{hwWO}_{3}$ and $\mathrm{hwWO} \mathrm{WO}_{2.5}$ derived from SE, was used as input to RCWA (see Fig. 5(a) and (b)). Fig. 6 compares the RCWA results with experimental data showing a very good agreement, both in the absolute value of reflection and the position of minimum and maximum. For comparison purposes the calculated reflectance from equally thick nonporous stoichiometric $\mathrm{hwWO}$ films on planar $\mathrm{Si}$ is also shown to show the clear deviation from our experimental data. In both cases the model of the non-porous stoichiometric $\mathrm{hwWO}_{x}$ is shifted towards higher wavelengths as expected. The reflection suppression of our coatings compared to the equally thick nonporous films is also evidenced by the reduction of the weighted to AM1.5 - average reflection $\left(R_{w}\right)$, within the region $400-900 \mathrm{~nm}$, which decreases from $22.2 \%$ to $13.6 \%$ and from $13.0 \%$ to $7.3 \%$ for the case of the $107 \mathrm{~nm}$ and $78 \mathrm{~nm}$ film, respectively.

\section{Conclusion}

Summarizing and concluding, it was shown that hot-wire metal oxide deposition is a simple method able to produce films exhibiting controllable in-depth profile of refractive index stemming from both the variation of stoichiometry and that of void fraction. The antireflective performance of thin and ultra-thin $\mathrm{hwWO}_{x}$ films on $\mathrm{Si}$ was presented and a significant reflection suppression, below $10 \%$ within the range of $500-1000 \mathrm{~nm}$, was shown for AOIs up to more than $60^{\circ}$. The deposition method allows for multiple switching of deposition ambient between $\mathrm{N}_{2}$ and $\mathrm{H}_{2}$ during the same run permitting further optimization of anti-reflection performance. SE analysis was employed to analyze the hwWO $\mathrm{W}_{x}$ optical properties layer by layer to extract the graded refractive index profile, which was subsequently used in the RCWA model to simulate the reflection properties. Our approach to simulate the reflection does not assume a specific model for the geometry of the surface, such as parabolic-, cone-like etc. The comparison between experimental and RCWA modeling results shows very good agreement. Film porosity along with the refractive index variations at the interfaces improves the omnidirectionality and broadband characteristics compared to the equally thick non-porous stoichiometric $\mathrm{WO}_{3}$.

\section{Acknowledgements}

N. Vourdas was financially supported by the State Scholarships Foundation of Greece-Post Doctoral fellowship program. ThetaMetrisis S.A. is acknowledged for providing the optical reflection measurement setup (FR-Basic).

\section{References}

[1] W. Guter, J. Schöne, S.P. Philipps, M. Steiner, G. Siefer, A. Wekkeli, E. Welser, E. Oliva, A.W. Bett, F. Dimroth, Applied Physics Letters 94 (2009).

[2] D.S. Hobbs, B.D. MacLeod, J.R. Riccobono, Proceedings of SPIE-The International Society for Optical Engineering 2007.

[3] R.R. King, D.C. Law, K.M. Edmondson, C.M. Fetzer, G.S. Kinsey, H. Yoon, D.D. Krut, J.H. Ermer, R.A. Sherif, N.H. Karam, Advances in Opto Electronics (2007).

[4] J.Q. Xi, M.F. Schubert, J.K. Kim, E.F. Schubert, M. Chen, S.Y. Lin, W. Liu, J.A. Smart, Nature Photonics 1 (2007) 176.

[5] Y. Li, J. Zhang, S. Zhu, H. Dong, F. Jia, Z. Wang, Y. Tang, L. Zhang, S. Zhang, B. Yang, Langmuir 26 (2010) 9842.

[6] Y. Du, L.E. Luna, W.S. Tan, M.F. Rubner, R.E. Cohen, ACS Nano 4 (2010) 4308.

[7] S.A. Boden, D.M. Bagnall, Applied Physics Letters 93 (2008).

[8] S. Chhajed, M.F. Schubert, J.K. Kim, E.F. Schubert, Applied Physics Letters 93 (2008).

[9] N.E. Vourdas, M.E. Vlachopoulou, A. Tserepi, E. Gogolides, International Journal of Nanotechnology 6 (2009) 196

[10] K. Choi, S.H. Park, Y.M. Song, Y.T. Lee, C.K. Hwangbo, H. Yang, H.S. Lee, Advanced Materials 22 (2010) 3713.

[11] B.S. Richards, Progress in Photovoltaics: Research and Applications 12 (2004) 253.

[12] G. Papadimitropoulos, N. Vourdas, K. Giannakopoulos, M. Vasilopoulou, D. Davazoglou, Journal of Applied Physics 109 (2011) 103527.

[13] M. Vasilopoulou, G. Papadimitropoulos, L.C. Palilis, D.G. Georgiadou, P. Argitis, S. Kennou, I. Kostis, N. Vourdas, N.A. Stathopoulos, D. Davazoglou, Organic Electronics 13 (2012) 796-806.

[14] M. Vasilopoulou, L.C. Palilis, D.G. Georgiadou, A.M. Douvas, P. Argitis, S. Kennou, L. Sygellou, G. Papadimitropoulos, I. Kostis, N.A. Stathopoulos, D. Davazoglou, Advanced Functional Materials 21 (2011) 1489.

[15] M. Vasilopoulou, L.C. Palilis, D.G. Georgiadou, P. Argitis, S. Kennou, L. Sygellou, I. Kostis, G. Papadimitropoulos, N. Konofaos, A.A. Iliadis, D. Davazoglou, Applied Physics Letters 98 (2011) 123301. 
[16] N. Vourdas, G. Papadimitropoulos, I. Kostis, M. Vasilopoulou, D. Davazoglou, Thin Solid Films 520 (2012) 3614-3619.

[17] D. Barreca, S. Bozza, G. Carta, G. Rossetto, E. Tondello, P. Zanella, Surface Science (2003) 439-443, 532-535.

[18] M.J. Son, S. Kim, S. Kwon, J.W. Kim, Organic Electronics 10 (2009) 637.

[19] Y.J. Cho, Y.W. Lee, H.M. Cho, I.W. Lee, S.Y. Kim, Journal of Applied Physics 85 (1999) 1114

[20] C.H. Chang, Y.P. Peichen, C.S. Yang, Applied Physics Letters 94 (2009).
[21] M.F. Saenger, J. Sun, M. Schädel, J. Hilfiker, M. Schubert, J.A. Woollam, Thin Solid Films 518 (2010) 1830.

[22] D.G. Stavenga, S. Foletti, G. Palasantzas, K. Arikawa, Proceedings of the Royal Society B: Biological Sciences 273 (2006) 661.

[23] K.C. Sahoo, Y. Li, E.Y. Chang, IEEE Transactions on Electron Devices 57 (2010) 2427

[24] M.G. Moharam, E.B. Grann, D.A. Pommet, T.K. Gaylor, Journal of the Optical Society of America A: Optics and Image Science, and Vision 12 (1995) 1068 